\title{
Inhibition of FK506 Binding Proteins Reduces $\alpha$-Synuclein Aggregation and Parkinson's Disease-Like Pathology
}

\author{
Melanie Gerard, ${ }^{1,4}$ Angélique Deleersnijder, ${ }^{1,5}$ Veronique Daniëls, ${ }^{5}$ Sarah Schreurs, ${ }^{3}$ Sebastian Munck, ${ }^{6}$ \\ Veerle Reumers, ${ }^{5}$ Hans Pottel, ${ }^{2}$ Yves Engelborghs, ${ }^{3}$ Chris Van den Haute, ${ }^{5}$ Jean-Marc Taymans, ${ }^{5}$ Zeger Debyser, ${ }^{1,4}$ \\ and Veerle Baekelandt ${ }^{5}$ \\ Laboratories of ${ }^{1}$ Biochemistry and ${ }^{2}$ Biophysics, Interdisciplinary Research Centre, Katholieke Universiteit Leuven-Kortrijk, B-8500 Kortrijk, Flanders, \\ Belgium, ${ }^{3}$ Laboratory of Biomolecular Dynamics, Katholieke Universiteit Leuven, B-3001 Leuven, Flanders, Belgium, and Laboratories of ${ }^{4}$ Molecular \\ Virology and Gene Therapy and ${ }^{5}$ Neurobiology and Gene Therapy and ${ }^{6}$ Flanders Institute for Biotechnology Department of Developmental and Molecular \\ Genetics, Katholieke Universiteit Leuven, B-3000 Leuven, Flanders, Belgium
}

$\alpha$-Synuclein ( $\alpha$-SYN) is a key player in the pathogenesis of Parkinson's disease (PD). In pathological conditions, the protein is present in a fibrillar, aggregated form inside cytoplasmic inclusions called Lewy bodies. Members of the FK506 binding protein (FKBP) family are peptidyl-prolyl isomerases that were shown recently to accelerate the aggregation of $\alpha$-SYN in vitro. We now established a neuronal cell culture model for synucleinopathy based on oxidative stress-induced $\alpha$-SYN aggregation and apoptosis. Using high-content analysis, we examined the role of FKBPs in aggregation and apoptotic cell death. FK506, a specific inhibitor of this family of proteins, inhibited $\alpha$-SYN aggregation and neuronal cell death in this synucleinopathy model dose dependently. Knockdown of FKBP12 or FKBP52 reduced the number of $\alpha$-SYN aggregates and protected against cell death, whereas overexpression of FKBP12 or FKBP52 accelerated both aggregation of $\alpha$-SYN and cell death. Thus, FK506 likely targets FKBP members in the cell culture model. Furthermore, oral administration of FK506 after viral vector-mediated overexpression of $\alpha$-SYN in adult mouse brain significantly reduced $\alpha$-SYN aggregate formation and neuronal cell death. Our data explain previously described neuroregenerative and neuroprotective effects of immunophilin ligands and validate FKBPs as a novel drug target for the causative treatment of PD.

\section{Introduction}

Parkinson's disease (PD) is characterized by the degeneration of dopaminergic neurons in the substantia nigra. One of the key proteins in PD is $\alpha$-synuclein ( $\alpha$-SYN) (for review, see Surguchov, 2008). In the dopaminergic neurons of PD patients, a fibrillar form of $\alpha$-SYN constitutes the main protein component of the characteristic cytoplasmic aggregates called Lewy bodies (LBs). Moreover, mutations in $\alpha$-SYN that are responsible for rare inherited forms of PD (point mutations or gene duplications/triplications) accelerate the aggregation of the protein.

$\alpha$-SYN is a small, intrinsically disordered protein that aggregates into a fibrillar $\beta$-pleated structure under pathological conditions. The central hydrophobic NAC domain confers its propensity for aggregation, whereas the highly charged hydrophilic C-terminal part counteracts self-aggregation, probably by shielding the NAC domain from the surrounding solution (Hoyer et al., 2004; Bertoncini et al., 2005).

\footnotetext{
Received Dec. 3, 2009; accepted Dec. 24, 2009

This work was supported by the Institute for the Promotion of Innovation by Science and Technology in Flanders (Projects SBO 30238, 30239, and 80020, a postdoctoral fellowship to M.G., and doctoral fellowship to A.D. and S.S.), the Fund for Scientific Research Fonds Wetenschappelijk Onderzoek Vlaanderen (Grant G.0406.06, a postdoctoral fellowship to J.-M.T., and doctoral fellowship to V.D.), and Katholieke Universiteit Leuven Grants 0T/08/052A and IOF-KP/07/001.

Correspondence should be addressed to Veerle Baekelandt, Laboratory of Neurobiology and Gene Therapy, Katholieke Universiteit Leuven, Kapucijnenvoer 33, B-3000 Leuven, Flanders, Belgium. E-mail: veerle.baekelandt@ med.kuleuven.be.

D0I:10.1523/JNEUROSC1.5983-09.2010

Copyright $\odot 2010$ the authors $\quad 0270-6474 / 10 / 302454-10 \$ 15.00 / 0$
}

We have shown that enzymes of the FK506 binding protein (FKBP) family accelerate the aggregation of recombinant $\alpha$-SYN in vitro and that FK506, a specific FKBP inhibitor, abrogates this effect (Gerard et al., 2006, 2008). FKBPs are members of the immunophilins, enzymes with a peptidyl-prolyl cis-trans isomerase (PPIase) activity that bind to immunosuppressants such as FK506 (Göthel and Marahiel, 1999). The human FKBP family counts 15 principal members with many different functions (Galat, 2003; Rulten et al., 2006). Four members of this family, FKBP12, FKBP38, FKBP52, and FKBP65, are enriched in the human brain (Steiner et al., 1992; Charters et al., 1994a,b; Coss et al., 1998).

Recently, neuroregenerative and neuroprotective properties have been assigned to FK506 and related compounds in a number of neurological disease models for Alzheimer's and Parkinson's diseases, as well as in more general neuropathy models (Gold et al., 1997, 2004; Avramut et al., 2001; Guo et al., 2001). At first, the immunosuppressive properties of FK506 were thought to be responsible for these properties. Later however, several synthetic ligands without immunosuppressive capacity were developed with equal or even stronger neuroprotective and regenerative effects, ruling out this possibility (Gold et al., 1997, 2004; Steiner et al., 1997; Guo et al., 2001; Edlich et al., 2006). Several studies have tried to identify the FKBP member involved and to elucidate the mechanism-of-action (Gold et al., 1997, 1999; Steiner et al., 1997; Kato et al., 2000; Guo et al., 2001; Avramut and Achim, 2002; Brecht et al., 2003; Davies et al., 2005; Edlich et al., 2006; 
Labrande et al., 2006; Woods et al., 2007). No conclusive answer has been provided so far, but the data suggest that PPIases may play a role in neurodegeneration (Gold et al., 1999; Avramut and Achim, 2002; Edlich et al., 2006; Labrande et al., 2006; Woods et al., 2007).

To investigate the physiological relevance of our previous finding, we studied the effect of FK506, FKBP12, and FKBP52 on the aggregation and neurotoxicity of $\alpha$-SYN in neuronal cell culture and mouse brain. Our data demonstrate that either inhibition by FK506 or downregulation of endogenous FKBP12 or FKBP52 reduces $\alpha$-SYN aggregation and concomitant neuronal cell death in cell culture and mouse brain. All data together validate FKBP as novel therapeutic drug target for PD.

\section{Materials and Methods}

Animals. All animal experiments were approved by the bioethical committee of the Katholieke Universiteit Leuven. We used adult C57BL/6 mice. Thy $1-\mathrm{A} 30 \mathrm{P}-\alpha \mathrm{SYN}$ transgenic mice were kindly provided by Dr. P. Kahle (Hertie Institute for Clinical Brain Research, University of Tübingen, Tübingen, Germany) The animals were housed under 14/10 h light/dark cycle with access to food and water ad libitum.

Cell culture. Human neuroblastoma SHSY5Y cells were grown in DMEM (Invitrogen) supplemented with 15\% fetal calf serum (International Medical), $500 \mu \mathrm{g} / \mathrm{ml}$ gentamycin (Invitrogen), and $1 \%$ nonessential amino acids (Invitrogen) (referred to herein as DMEM-complete) at $37^{\circ} \mathrm{C}$ and $5 \% \mathrm{CO}_{2}$ in a humidified atmosphere.

Generation of stable overexpression cell lines using lentiviral vectors. Lentiviral vector (LV) constructs encoding human $\alpha$-SYN, luciferase, FKBP12, or FKBP52 under control of the cytomegalovirus (CMV) promoter were prepared in house as described previously (Geraerts et al., 2005). LV constructs allowed coexpression of the puromycin selection marker from the same transcript via an internal ribosome entry site. For transduction and selection of cells in culture, 500,000 SHSY5Y cells were plated in a six-well plate and grown in DMEM-complete. The next day, vector was applied to SHSY5Y cells for $16 \mathrm{~h}$, after which the vectorcontaining medium was replaced by DMEM-complete with $1 \mu \mathrm{g} / \mathrm{ml}$ puromycin. After $5 \mathrm{~d}$ of selection, cells were controlled for overexpression of specific proteins via Western blot. Using separate selection markers per gene, we also generated SHSY5Y cell lines overexpressing both $\alpha$-SYN and FKBP12 or FKBP52.

Transient knockdown of FKBP12 and FKBP52. Two short interfering RNA (siRNA) molecules were designed for each FKBP. For human FKBP12 (Entrez Gene identification number 2280), siFKBP12_86 starts at base 86 of the cDNA sequence (target sequence, GGATGCTTGAAGATGGAAATT), and siFKBP12_121 starts at base 121 (target sequence, GGGACAGAAACAAGCCCTTTA). For FKBP52 (Entrez Gene identification number 2288), siFKBP52_446 starts at base 446 of its cDNA sequence (target sequence, GAATCATTCGCAGAATACATT), and siFKBP52_1023 starts at base 1023 (target sequence, CTGTAACAAGGCCCTAGAATT). All siRNA molecules were purchased from Qiagen. A total of 125,000 SHSY5Y cells were plated in a 24-well plate. The following day, the cells were transfected with the respective siRNAs using siFECTamine (IC-Vec) as a transfection reagent. Mock transfected cells, exposed to transfection reagent only, were used as a control. Per well, $0.86 \mu \mathrm{l}$ of siRNA $(200 \mu \mathrm{M})$ was mixed with $221 \mu \mathrm{l}$ of Opti-MEM (Invitrogen) without additions and $9.6 \mu \mathrm{l}$ of siFECTamine. This solution was vortexed and incubated at room temperature for $5 \mathrm{~min}$. Optimem (100 $\mu \mathrm{l})$ was applied to the cells after a washing step with Optimem. Next, the siRNA mixture was added to the well. The cells were incubated with this transfection medium for $6 \mathrm{~h}$. For induction of aggregation, the medium was then replaced with DMEM-complete containing $10 \mathrm{mM} \mathrm{FeCl}_{2}$ and $100 \mu \mathrm{M} \mathrm{H} \mathrm{H}_{2} \mathrm{O}_{2}$, freshly prepared, and filtered through a $0.22 \mu \mathrm{m}$ filter. After $3 \mathrm{~d}$, cells were fixed and aggregates were visualized with Thioflavin $S$ (Thios).

Stable knockdown of FKBP12. The sequence of siFKBP12_86 was cloned in pCHMWS-mU6-MCS-CMV-Hygro (MCS indicates multiple cloning site), an LV transfer plasmid optimized for the production of short hairpin RNA (shRNA) (Van den Haute et al., 2003). A mouse U6 promotor directed transcription of the hairpin sequence $5^{\prime}$-cta GGA TGC TTG AAG ATG GAA ATT CTTCCTGTCA AAT TTC CAT CTT CAA GCA TCC ttttt ggaa-3' (sense and antisense sequence of siFKBP12_86 are given in bold, and italics show the miR23 loop). For the mismatch sequence used as a negative control, base pairs $9-12$ of the siFKBP12_86 sequence were complementary to the active sequence, thus producing an inactive shRNA hairpin. The plasmid also contained a hygromycin resistance gene under control of a CMV promotor. The triple transfection production method was then used to generate LV expressing shFKBP12_86 and shFKBP12_86_MM (further mentioned as shFKBP12 and shFKBP12_MM). After transduction of SHSY5Y cells with these LVs and selection with hygromycin, the expression of FKBP12 was verified via Western blot. All cell lines (overexpression and knockdown lines) were kept in culture no longer than 10 passages to avoid loss of cells with strongest knockdown.

Western blotting. Cells were plated in 24 -well plates $(150,000$ cells per well). The next day, cells were washed twice with PBS and lysed with 100 $\mu \mathrm{l}$ of lysis solution [20 mM HEPES, pH 7.4, 1\% SDS, protease cocktail inhibitor (diluted as suggested by the manufacturer; Roche Diagnostics), and $0.5 \mathrm{~mm}$ EDTA]. Whole-cell extracts were boiled for $3 \mathrm{~min}$ and homogenized by eight passages through a 30 gauge insulin syringe, followed by a final boiling step for $1 \mathrm{~min}$. Extract from each cell containing $10 \mu \mathrm{g}$ of total protein (measured by BCA protein assay kit; Fisher Thermo Scientific) was separated by SDS-PAGE (16\% polyacrylamide) and electroblotted for $2 \mathrm{~h}$ at $30 \mathrm{~V}$ onto polyvinylidene difluoride membranes (Bio-Rad). Membranes were blocked with 5\% milk powder in PBS supplemented with $0.1 \%$ Tween 20 , and detection was performed using specific antibodies against FKBP12 (rabbit polyclonal antibody, 1:5000 dilution; Affinity BioReagents), FKBP52 (mouse monoclonal antibody, 1:2000 dilution; Stressgen), $\alpha$-SYN (rabbit polyclonal antibody, 1:5000 dilution; Millipore Bioscience Research Reagents), or luciferase (goat polyclonal antibody, 1:1000 dilution; Promega). Glyceraldehyde-3phosphate dehydrogenase (GAPDH) antibody (rabbit polyclonal antibody, 1:1000 dilution; Abcam) was used to control for equal loading. Detection was performed using chemiluminescence $\left(\mathrm{ECL}^{+}\right.$-kit; GE Healthcare) and a horseradish peroxidase (HRP)-conjugated secondary antibody (Dako). Alternatively, $\alpha$-tubulin was used as a loading control. In this case, the membrane was washed overnight with PBS plus $0.1 \%$ Tween 20 , followed by a $30 \mathrm{~min}$ incubation with a mouse anti- $\alpha$-tubulin antibody (1:10,000 dilution; Sigma-Aldrich). HRP-conjugated goat antimouse (Dako) was used as a secondary antibody.

Immunocytochemistry. For immunocytochemistry, cells were grown on 12-mm-diameter round glass plates (Menzel-Gläser) within the wells of a 24-well plate. After an initial wash step with PBS, cells were fixed with $4 \%$ formaldehyde for $15 \mathrm{~min}$. After a $30 \mathrm{~min}$ blocking step with $2 \%$ BSA in PBS, cells were incubated overnight with primary antibody (1:2000 anti- $\alpha$-SYN and 1:2000 anti-FKBP12 or 1:500 anti-FKBP52) in PBS plus $1 \%$ BSA. After washing twice with PBS for $15 \mathrm{~min}$, the cells were incubated for $2 \mathrm{~h}$ with secondary antibody (1:2000 AlexaFluor 488- or 633conjugated antibodies; Invitrogen) and washed again with PBS twice for $15 \mathrm{~min}$. Thereafter, the glass plates were mounted on a microscope slide with Mowiol solution (Sigma). Fluorescence was detected with the 488 argon-ion or the 633 helium-neon laser with a laser scanning microscopy unit (LSM 510; Carl Zeiss).

Cell culture model for induction of $\alpha$-SYN aggregation. To induce $\alpha$-SYN aggregation, the cell culture model described by Ostrerova-Golts et al. (2000) was used with minor modifications. Briefly, 150,000 cells were plated on round glass plates in a 24 -well plate, or, alternatively for high-content analysis, 150,000 cells per well were plated in a 48 -well plate (Greiner Bio-One). The next day, cells were treated for $72 \mathrm{~h}$ with $100 \mu \mathrm{M}$ $\mathrm{H}_{2} \mathrm{O}_{2}$ and $7.5 \mathrm{~mm}$ freshly prepared $\mathrm{FeCl}_{2}$ in DMEM-complete and filtered through a $0.22 \mu \mathrm{m}$ filter. Different concentrations of compounds (a stock solution in DMSO was diluted 100-fold) were added to this stressinducing medium. The control condition received a similar amount of DMSO. Thereafter, cells were fixed for $15 \mathrm{~min}$ in $4 \%$ formaldehyde. After two PBS washes, cells were incubated with $0.05 \%$ ThioS (Sigma) for 20 min, washed twice in $80 \%$ ethanol, and washed once in PBS. For immunocytochemical (ICC) staining, the procedure was as described in that 
section. For manual scoring, the glass plates were mounted with Mowiol solution (Sigma). For high-content analysis, a $0.1 \mu \mathrm{g} / \mathrm{ml} 4^{\prime}, 6^{\prime}$-diamidino-2phenylindole (DAPI) solution in PBS was applied to the cells, which were stored at $4^{\circ} \mathrm{C}$ until analysis.

Manual counting of $\alpha$-SYN aggregation. Intracellular fibrillar $\alpha$-SYN aggregates were detected after ThioS or ICC staining (using a Alexa 488conjugated secondary antibody) with a 488 argon-ion laser. For each condition, at least eight independent samples (glass plates) were assayed ( $8 \leq n \leq 16$ for all experiments). Of each sample, three confocal images were taken with an inverted confocal fluorescence microscope (LSM 510 Meta; Carl Zeiss) after staining, and $\sim 100$ cells were determined to be aggregate positive or negative (for an example of aggregate positive and negative cells, see Fig. 1A,B). The conditions of the experiment were blinded to the investigator scoring the cells.

High-content analysis. To automate the aggregation assay, the IN Cell Analyzer 1000 (GE Healthcare) was used. This instrument generates fluorescence microscopy images of cell samples in a multiwell plate format in an automated manner, followed by automated image analysis. For each condition, eight independent samples (wells) were assayed, which resulted in $\sim 1000-5000$ analyzed cells per well. The nucleus of each cell and possible apoptotic inclusions were detected via the DAPI staining (xenon lamp, excitation filter at $360 \mathrm{~nm}$ and emission filter at $460 \mathrm{~nm}$ ). The cytoplasm and possible fibrillar $\alpha$-SYN aggregates within were detected via the ThioS staining (excitation at $480 \mathrm{~nm}$, emission at $535 \mathrm{~nm}$ ). Images were analyzed using IN Cell Investigator software. The cytoplasm was identified using the background fluorescence of the ThioS staining. In the cytoplasm, $\alpha$-SYN inclusions were detected based on increased fluorescence compared with the background intensity. As a measure for cell death, the appearance of bright nuclear inclusions pointing to nucleus condensation and fragmentation was chosen.

Stable overexpression of $\alpha-S Y N$ in the striatum of C57BL/6 mice. To assess the possible effect of immunophilin ligands on the aggregation of $\alpha$-SYN in vivo, the $\alpha$-SYN mouse model of Lauwers et al. (2003) was used. Briefly, $2 \mu \mathrm{l}$ of lentiviral vector encoding $\alpha$-SYN was stereotactically injected in the striatum of 11 -week-old C57BL/6 female mice. The mice were divided into one control group and three FK506 treatment groups. Long plasma half-life of FK506 (>20 h) (Sakai et al., 2004) and linear pharmacokinetic profile (cytochrome P450 3A4 metabolized) allow for once daily administration. FK506 has also been shown to cross the blood-brain barrier and to decrease PPIase activity in the brain of rats (Brecht et al., 2003; Murakami et al., 2004). Treatment with FK506 was initiated 1 week after surgery. We included a high functionally active dose (group $4 ; 8 \mathrm{mg} \cdot \mathrm{kg}^{-1} \cdot \mathrm{d}^{-1}, n=6$ ), a lower functionally active dose (group $3 ; 2 \mathrm{mg} \cdot \mathrm{kg}^{-1} \cdot \mathrm{d}^{-1}, n=7$ ), and a borderline/possibly inactive dose (group 2; $0.5 \mathrm{mg} \cdot \mathrm{kg}^{-1} \cdot \mathrm{d}^{-1}, n=6$ ). FK506 was dissolved in suitable vehicle (1\% Cremophor EL, $4 \%$ ethanol) and administered in volumes of $10 \mu \mathrm{l} / \mathrm{g}$ body weight by oral gavages once daily. Control animals in group 1 were administrated the vehicle $(n=6)$. FK506 (Prograf) was purchased from Fujisawa Healthcare. Capsules were opened and the content was dissolved in the vehicle solution in concentrations of $0.05,0.2$, and $0.8 \mathrm{mg} / \mathrm{ml}$. Treatment was performed daily for 5 months. No significant negative effects of the FK506 treatment were observed, except for a somewhat slower weight gain in all groups (vehicle- and FK506-treated mice).

Perfusion and immunohistochemistry. After 5 months, animals were perfused transcardially and the brain was fixed in $4 \%$ paraformaldehyde overnight. For further storage, brains were put in PBS with $0.1 \%$ sodium azide. All brains were sliced in $50 \mu \mathrm{m}$ coronal slices with a Leica VT $1000 \mathrm{~S}$ vibratome. Immunohistochemical staining was performed on every fifth section of the striatum. Briefly, sections were exposed to $3 \%$ hydrogen peroxide and incubated overnight with a polyclonal rabbit anti- $\alpha$-SYN (1:5000; Millipore Bioscience Research Reagents) in 10\% normal swine serum. For a fluorescent staining, the secondary antibody (1:250 dilution of AlexaFluor 488-conjugated goat anti-rabbit antibody; Invitrogen) was applied for $1 \mathrm{~h}$ after three wash steps (one quick wash step, two $5 \mathrm{~min}$ wash steps). For a visual staining, the wash steps were followed by an incubation with biotinylated secondary antibody (Dako), after which the streptomycin-ABC-HRP complex (Dako) was applied. Chromogenic detection was performed using diaminobenzidine as a substrate.
For the colocalization study between FKBP12 and $\alpha$-SYN inclusions, 19 -month-old transgenic mice were used (Thy1-A30P- $\alpha$-SYN). These were provided by Dr. Philipp Kahle and described previously by Freichel et al. (2007).

Analysis of $\alpha-S Y N$ expression and aggregation in vivo. Stained brain sections were analyzed in two ways. For each analysis, the conditions of the experiment were blinded to the investigator. First, the volume of the brain area transduced with $\alpha$-SYN was quantified by a stereological procedure based on the Cavalieri principle (Taymans et al., 2007). For each animal, serial sections (minimum five) with an interval of $500 \mu \mathrm{m}$ centering around the injection site were analyzed by means of a Bioquant Image Analyzing System ( $R \&$ M Biometrics) connected with a CCD video camera to the microscope. A point-counting grid was placed over the screen, on which the entire transduced brain region was displayed from a low-power objective. An example of such a region is shown in Figure $6 B$. Points overlying $\alpha$-SYN-positive cells and fibers were counted. The transduced volume was calculated by multiplying the sum of the counted points with the distance between the counted sections and the area associated with each point on the grid.

As second means of analysis, we determined the number of $\alpha$-SYNpositive cells exhibiting LB-like aggregates in the cytoplasm. Therefore, slices were investigated with a $100 \times$ magnifying lens. Fifty neurons overexpressing $\alpha$-SYN were counted, and each neuron was visually scored for the presence or absence of aggregates. An example of an $\alpha$-SYN-positive cell with and without aggregates is displayed in the inlay image of Figure $6 B$.

Statistical analysis. All statistical analyses were performed in Prism 5.0 (GraphPad Software). For multiple group comparisons, ANOVA followed by a post hoc test (Dunnett's test) to correct for multiple testing was used. In case of non-normality, the nonparametric equivalent (Kruskal-Wallis test) was chosen, followed by Dunn's test. A simple $t$ test or Mann-Whitney $U$ test (non-normality) was used in case only two groups were compared. $p$ values are indicated as follows: ${ }^{*} p<0.05,{ }^{* *} p<0.01,{ }^{* * *} p<0.001$.

\section{Results}

\section{Development of a novel cell culture model}

for synucleinopathy

To induce $\alpha$-SYN aggregation in a cell culture model, we exposed SHSY5Y neuroblastoma cells to oxidative stress conditions using $\mathrm{H}_{2} \mathrm{O}_{2}$ and $\mathrm{FeCl}_{2}$ according to a protocol adapted from OstrerovaGolts et al. (2000). After 3 d of continued oxidative stress, cytoplasmic $\alpha$-synuclein inclusions could be visualized using either an immunocytochemical staining for $\alpha$-SYN or a ThioS staining, demonstrating the fibrillar nature of the aggregates (Fig. $1 A, B$ ). Cytoplasmic aggregates of endogenous $\alpha$-SYN were induced in $\sim 8 \%$ of the cells. In stable $\alpha$-SYN-overexpressing SHSY5Y cells, the number of cells with $\alpha$-SYN inclusions increased to $\sim 35 \%$ under the same conditions (Fig. 1E). Overexpression of a control PD-unrelated protein (luciferase) did not affect $\alpha$-SYN aggregation.

\section{High-content analysis of $\alpha$-synuclein aggregation and apoptosis}

To facilitate the quantitative analysis of $\alpha$-SYN inclusion formation in our cell culture model, we developed a high-content multiparametric method. Both wild-type (WT) SHSY5Y cells and $\alpha$-SYN-SHSY5Y cells were analyzed with our automated image acquisition and analysis protocol (cf. Materials and Methods) after $72 \mathrm{~h}$ of oxidative stress treatment. For each cell line, eight independent samples were measured (eight wells of a 48 -well plate) and, in each well, an average of 500-1000 cells were visualized and analyzed. Each cell was identified via DAPI nuclear staining (Fig. $1 D$, left, blue contours). The cytoplasm was determined based on the background ThioS staining (Fig. $1 D$, middle, green contours). Inclusion formation was detected based on the presence of high-intensity spots in the cytoplasm after ThioS staining (Fig. 1D, middle, yellow contours) or immunocytochemical staining with an antibody against $\alpha$-SYN (Fig. $1 C$, middle). The 

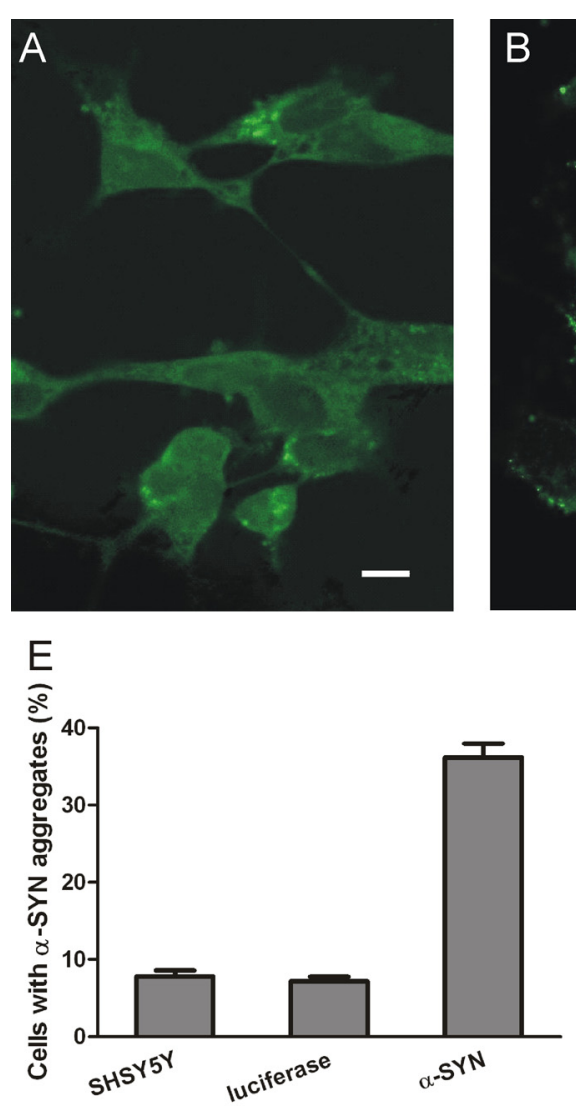

\section{$\mathrm{F}$}

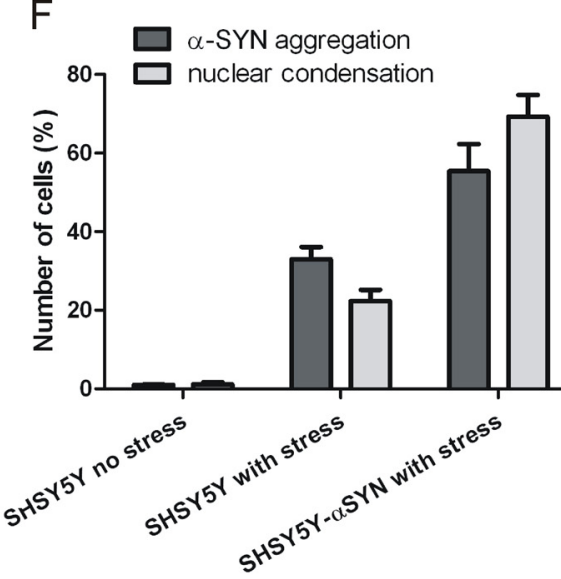

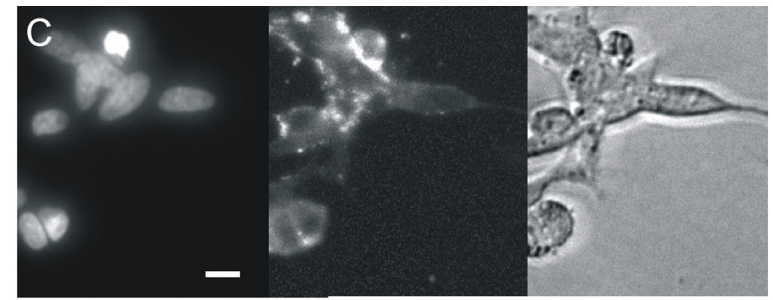

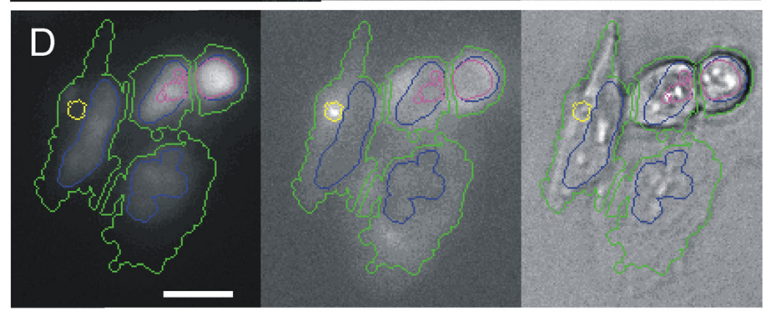

G

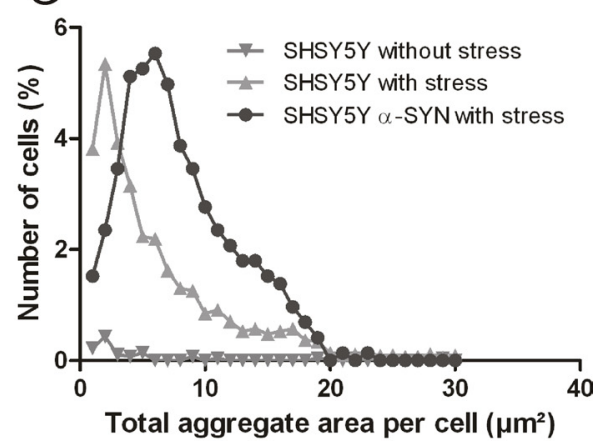

Figure 1. Induction of $\alpha$-SYN aggregation and apoptosis in SHSY5Y neuroblastoma cells by oxidative stress. $\boldsymbol{A}, \boldsymbol{B}$, Thio staining $(\boldsymbol{A})$ and immunocytochemical staining $(\boldsymbol{B})$ of intracytoplasmic $\alpha$-SYN aggregates in SHSY5Y cells after induction of oxidative stress. Scale bars, $10 \mu \mathrm{m}$. These images are used for manual quantification of aggregation. $\boldsymbol{C}, \boldsymbol{D}$, Pictures taken with the IN cell analyzer for high-content analysis. C, Example of nucleus detection via DAPI staining (left) and $\alpha$-SYN detection via immunocytochemical staining (middle). D, Example of nucleus detection via DAPI staining (left) and $\alpha$-SYN fibril detection via ThioS staining (middle). The cell nucleus is identified based on the DAPI fluorescence (blue) and the cytoplasm via the ThioS background fluorescence (green). Intracytoplasmic $\alpha$-SYN inclusions (yellow) and nuclear apoptotic fragments (pink) are detected based on a high-intensity compared with the background staining in the cytoplasm and the nucleus, respectively. $\boldsymbol{E}$, Percentage of cells containing $\alpha$-SYN aggregates determined manually after induction of oxidative stress. Both wild-type SHSY5Y cells and $\alpha$-SYN-overexpressing cells were analyzed. A luciferase-0verexpressing cell line was used as a negative control. SEM is shown for each condition. $\boldsymbol{F}$, High-content analysis determining percentage of cells with (1) $\alpha$-SYN aggregates and (2) nuclear condensation in wild-type SHSY5Y cells with and without stress and in $\alpha$-SYN-overexpressing SHSY5Y cells with stress. SEM is shown for each condition. G, Histogram of the area distribution of $\alpha$-SYN aggregates per cell in the cells containing aggregates (high-content analysis). The percentage of cells with $\alpha$-SYN aggregates for these cell lines is shown in $\boldsymbol{F}$.

relative results between different conditions were similar for the immunocytochemical staining and the ThioS staining. Because of biological relevance (detection of amyloid aggregates) and faster handling time, only ThioS high-content results are shown.

$\alpha$-SYN inclusion formation is strongly induced in conditions of oxidative stress as revealed by the total area of $\alpha$-SYN inclusions per cell (Fig. $1 G$ ) and the number of aggregate-positive cells (Fig. 1F). This effect is even more pronounced after overexpression of $\alpha$-SYN. Comparison of the absolute number of cells with $\alpha$-synuclein inclusions between both methods revealed a more sensitive detection with the high-content analysis compared with the manual quantification. In parallel, we quantified the number of highly fluorescent fragments in the nucleus as a measure of late apoptotic cells (Fig. $1 D$, left, pink contours). This parameter revealed a positive correlation between $\alpha$-SYN aggregation and apoptosis (Fig. $1 F$ ).

\section{FK506 reduces $\alpha$-SYN aggregation and apoptosis in the synucleinopathy model}

Next, we tested the effect of the inhibitor FK506 in our cell culture model for $\alpha$-SYN aggregation (Fig. 2). Manual determination of the percentage of aggregate-positive cells revealed a significant decrease in the number of $\alpha$-SYN inclusions in $\alpha$-SYNoverexpressing cell lines after FK506 treatment (Fig. 2 A). In WT SHSY5Y cells and in luciferase-overexpressing cells used as a negative control, no pronounced effect of FK506 was observed, probably because of low endogenous levels of $\alpha$-SYN (see Fig. 5D). To correlate $\alpha$-SYN aggregation with cell viability, we repeated this experiment using high-content analysis. This evidenced a dosedependent reduction in $\alpha$-SYN aggregation by FK506 treatment accompanied by a decrease in apoptosis (Fig. $2 B$ ).

\section{Knockdown of FKBP12 or FKBP52 reduces oxidative stress-induced $\alpha$-SYN aggregation and apoptosis}

To verify whether the observed effect of FK506 was attributable to a direct inhibition of one or different FKBPs, we decided to knock down FKBP12 or FKBP52 in SHSY5Y cells transiently with siRNA and stably using shRNA. First, SHSY5Y cells were transfected with siRNA molecules against FKBP12 (siFKBP12_86 and siFKBP12_121) or FKBP52 (siFKBP52_446 and siFKBP52_1023). At different time points after transfection, total cell extracts were made, and endogenous levels of FKBP12 or FKBP52 were visualized via Western blot. Both siFKBP12 sequences efficiently si- 
lenced FKBP12 expression for as long as $7 \mathrm{~d}$ (Fig. 3A) without affecting the expression of FKBP52 (Fig. 3B). Therefore, the time window of knockdown sufficiently exceeds the $3 \mathrm{~d}$ needed for the aggregation induction test. Conversely, for FKBP52, a better knockdown was obtained with the siFKBP52_1023 sequence than with siFKBP52_446 (Fig. 3C). Therefore, we decided to proceed only with siFKBP52 1023. Again, the knockdown lasted for $7 \mathrm{~d}$. Neither of the FKBP52 siRNA molecules affected FKBP12 expression (Fig. 3D). Consequently, SHSY5Y cells were transfected with siFKBP12_86, siFKBP12_121, or siFKBP52_ 1023 to knock down FKBP12 and FKBP52, respectively, followed by induction of oxidative stress (with a delayed onset of $6 \mathrm{~h}$ ). Stress was maintained for $72 \mathrm{~h}$. Quantification of the $\alpha$-SYN aggregates revealed that all three siRNAs reduced the number of fibrillar $\alpha$-SYN inclusions to $40-60 \%$ of the number in mock transfected SHSY5Y cells (Fig. 3E).

Because siFKBP12_86 induced the strongest effect on $\alpha$-SYN aggregation, we generated stable FKBP12 knockdown cell lines using an LV encoding an shRNA sequence derived from the siFKBP12_86 sequence (referred to as shFKBP12). As a negative control, we constructed an shFKBP12_86_mismatch LV (referred to as shFKBP12_MM) in which nucleotides 9-12 were modified to be noncomplementary to the target sequence. Because breakdown of the target mRNA normally starts here, noncomplementarity of these base pairs results in ineffective knockdown. Stable FKBP12 knockdown cell lines were generated with these vectors in WT SHSY5Y cells and $\alpha$-SYN-overexpressing SHSY5Y cells. Western blotting confirmed FKBP12 expression to be strongly reduced in the shFKBP12 cell line and unaffected in the shFKBP12_MM cell line (Fig. 4A). FKBP52 expression remained unaffected. We also verified that the expression of $\alpha$-SYN and FKBP12 did not change during oxidative stress conditions (data not shown).

In $\alpha$-SYN-overexpressing cells, high-content analysis revealed $\alpha$-SYN aggregation to be significantly inhibited by stable FKBP12 depletion compared with the control shFKBP12_MM cell line (Fig. $4 B$ ). The same trend was observed for SHSY5Y cells with endogenous levels of $\alpha$-SYN, but these differences were not statistically significant. Moreover, the number of late apoptotic cells was strongly reduced in the FKBP12 knockdown cell line compared with the negative control. Again, although the same trend was observed in WT SHSY5Y cells, the difference only reached statistical significance in the $\alpha$-SYN-overexpressing cells. If both FKBP12 and FKBP52 were simultaneously downregulated in $\alpha$-SYN-overexpressing cells, an additional 50\% decrease in $\alpha$-SYN aggregation and late apoptosis was observed (data not shown).

\section{Overexpression of FKBP12 and FKBP52 enhances $\alpha$-SYN aggregation and apoptosis in a cellular model for synucleinopathy}

Next we investigated whether overexpression of FKBP12 or FKBP52 resulted in enhanced $\alpha$-SYN aggregation. Stable FKBP-overexpressing SHSY5Y cells were generated by transduction with LV coding for FKBP12 or FKBP52. Overexpression of the transgenes was confirmed by Western blotting (Fig. $5 A$ ). The subcellular localization of overexpressed FKBP12 and
FKBP52 in SHSY5Y cells was determined by confocal microscopy under normal conditions and after induction of $\alpha$-SYN aggregates. FKBP12 primarily located in the cytoplasm (Fig. 5B), whereas FKBP52 was also mainly present in the cytoplasm with some nuclear staining (Fig. 5C). Aggregate formation did not alter subcellular FKBP localization (data not shown).

Next, we quantified the effect of FKBP12 and FKBP52 overexpression on $\alpha$-SYN aggregation after induction of oxidative stress $(48 \mathrm{~h})$ in the presence or absence of different concentrations of FK506. Manual counting revealed that overexpression of FKBP12, and to a somewhat lesser extent FKBP52, induced the formation of fibrillar $\alpha$-SYN inclusions (Fig. 5D), whereas overexpression of luciferase had no effect. The addition of FK506 to the medium resulted in a dose-dependent decrease of $\alpha$-SYN aggregation, indicating the specificity of the FKBP effect. Highcontent analysis was performed on cells overexpressing FKBP12. There was a clear increase in both the aggregation of $\alpha$-SYN and apoptotic nuclear fragmentation (Fig. 5E). This trend was observed in both WT SHSY5Y and $\alpha$-SYN-SHSY5Y cells, but again statistical significance was only reached in the $\alpha$-SYN-SHSY5Y cells.

FKBP12 colocalizes with $\alpha$-SYN inclusions in the brain of aged $\alpha$-SYN transgenic mice

To demonstrate a potential in vivo role of FKBP12 in $\alpha$-SYN aggregation, we performed immunohistochemical stainings on aged Thy1-A30P- $\alpha$-SYN transgenic mice (Freichel et al., 2007). At an age of 19 months, these mice display clear cytoplasmic $\alpha$-SYN inclusions in several brain regions, such as the deep mesencephalic nuclei (Fig. 6A). We observed a remarkable colocalization of FKBP12 within these inclusions.

FK506 treatment reduces $\alpha$-SYN aggregation and neurodegeneration in mouse brain

To verify whether FK506 inhibits $\alpha$-SYN aggregation in the brain as well, we tested the effect of oral administration of FK506 to C57BL/ 6 mice after stereotactic injection of $\alpha$-SYN-encoding LV in the striatum. We have shown previously that LV-mediated local overexpression of $\alpha$-SYN in rodent striatum or substantia nigra induces a time-dependent aggregation of $\alpha$-SYN and neurodegeneration (Lauwers et al., 2003, 2007). Two weeks after injection, three animals were killed to verify $\alpha$-SYN overexpres- 

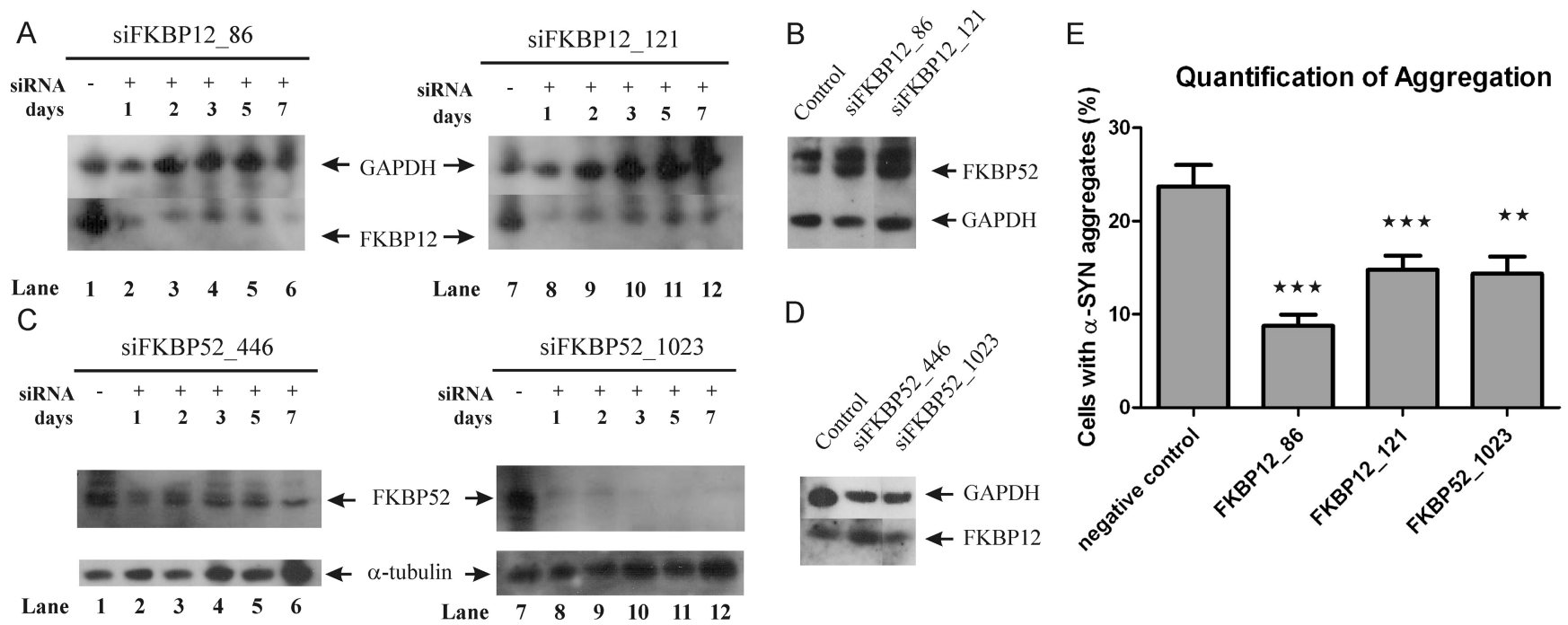

Figure 3. Transient FKBP knockdown inhibits $\alpha$-SYN aggregation and apoptosis in SHSY5Y cells. A, Western blot showing knockdown of FKBP12 in SHSY5Y cells. In each lane, $10 \mu \mathrm{g}$ of total protein was loaded, and FKBP12 expression was visualized by immunoblot. Lane 1, Extract of untreated SHSY5Y cells. Lanes 2-6, Cell extracts made 1, 2, 3, 5, and $7 \mathrm{~d}$ after transfection with siFKBP12_86. Lane 7, Extract of untreated SHSY5Y cells. Lanes $8-12$, Cell extracts made 1,2,3,5, and $7 \mathrm{~d}$ after transfection with siFKBP12_121. $\boldsymbol{B}$, Western blot of FKBP52 in the same cell extracts (3 d after transfection) with unaltered levels of FKBP52 after knockdown of FKBP12. GAPDH blotting controls for equal loading. C, Western blot showing FKBP52 knockdown in SHSY5Y cells. In each lane, cell extract containing $10 \mu \mathrm{g}$ of total protein was loaded and FKBP52 expression was visualized. Lane 1, Extract of untreated SHSY5Y cells. Lanes 2-6, Cell extracts made 1, 2, 3, 5, and $7 \mathrm{~d}$ after transfection with siFKBP52_446. Lane 7, Extract of untreated SHSY5Y cells. Lanes 8-12, Cell extracts made 1, 2, 3, 5, and $7 \mathrm{~d}$ after transfection with siFKBP52_1023. D, Western blot of FKBP12 in the same cell extracts ( $3 \mathrm{~d}$ after transfection) with unaltered levels of FKBP12 after knockdown of FKBP52. $\alpha$-Tubulin or GAPDH blotting confirm equal loading. $E$, After $6 \mathrm{~h}$ of incubation with respective siRNA molecules, oxidative stress was induced for 72h. SHSY5Y cells without siRNA treatment were used as a control. The percentage of ThioS-positive $\alpha$-SYN aggregates was determined. SEM is shown for each condition. $p$ values are indicated compared with control condition (mock transfection).
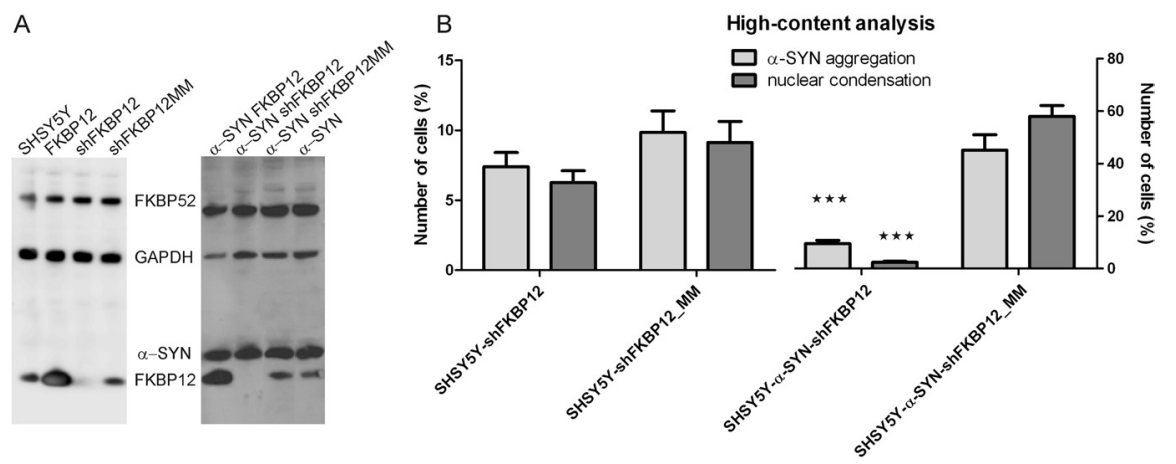

Figure 4. Stable FKBP12 knockdown inhibits $\alpha$-SYN aggregation and apoptosis in SHSY5Y cells. $A$, Left, Western blot showing stable FKBP12 knockdown in wild-type SHSY5Y cells after transduction with LV encoding shFKBP12 or shFKBP12_MM, followed by selection with hygromycin. In each lane, cell extract containing $10 \mu \mathrm{g}$ of total protein was loaded and FKBP12 expression was visualized. Lane 1, Untreated SHSY5Y cells. Lane 2, FKBP12-overexpressing SHSY5Y. Lane 3, Knockdown of endogenous FKBP12 by shFKBP12. Lane 4, SHSY5Y expressing shFKBP12_MM. GAPDH expression is shown as loading control. FKBP52 expression is unaffected by FKBP12 overexpression or knockdown. Right, Stable FKBP12 knockdown in SHSY5Y cells overexpressing $\alpha$-SYN. Lane 1, FKBP12-overexpressing $\alpha$-SYN-SHSY5Y. Lane 2, Knockdown of endogenous FKBP12. Lane 3, $\alpha$-SYN-SHSY5Y expressing shFKBP12_MM. Lane 4, $\alpha$-SYN-SHSY5Y. B, High-content analysis of $\alpha$-SYN aggregation and apoptosis in SHSY5Y cells and $\alpha$-SYN-SHSY5Y cells after knockdown of FKBP12. Error bars indicate the SEM. $p$ values are indicated compared with control condition (cell lines expressing shFKBP12_MM).

sion. In the three animals, similar expression levels of $\alpha$-SYN were detected in the striatum (Fig. $6 B, C$ ). The mice were then divided in four groups, receiving daily vehicle solution or three different doses of FK506.

After 5 months, all animals were killed, and the brains were analyzed by immunohistochemistry. Immunostainings for $\alpha$-SYN revealed inclusions in a subpopulation of the $\alpha$-SYNoverexpressing cells (Fig. $6 B, C$, inlay images). As reported previously, the inclusions were also ubiquitin positive (Fig. $6 C$ ), qualifying them as Lewy body-like inclusions.

Quantification of the proportion of $\alpha$-SYN-overexpressing cells with $\alpha$-SYN aggregates revealed that FK506 treatment signifi- cantly reduced the number of $\alpha$-SYN inclusions already at the lowest dose tested (Fig. $6 D)$. Increasing the dose of FK506 did not further decrease $\alpha$-SYN aggregation. Although no statistical difference was observed between groups receiving increasing concentrations of FK506 ( $p=$ 0.884 ), a highly significant difference was seen between the pooled groups of mice receiving FK506 treatment and the vehicle group $(p=0.0135)$.

We also investigated the effect of FK506 on $\alpha$-SYN-induced neuronal cell death. Therefore, we quantified the transduced brain volume by stereology as a measure for the number of $\alpha$-SYNoverexpressing cells, as described previously (Lauwers et al., 2003). Significantly more $\alpha$-SYN-positive cells survived when mice were treated with FK506 compared with vehicle solution ( $p=0.0043$ ) (Fig. $6 D)$. Again, no significant difference was observed between animals receiving increasing concentrations of FK506 ( $p=0.456)$.

\section{Discussion}

We developed a high-content neuronal cell assay to measure oxidative stress-induced $\alpha$-SYN aggregation and apoptosis. We compared these results with a manual analysis of $\alpha$-SYN aggregation. Using both methods, we demonstrated that FK506 treatment reduces $\alpha$-SYN aggregation and apoptosis in SHSY5Y cells. This result could be mimicked by knockdown of FKBP12 or FKBP52, suggesting that FK506 mediates neuroprotection through FKBP members. Conversely, overexpression of FKBP12 or FKBP52 significantly enhanced $\alpha$-SYN aggregation and cell 


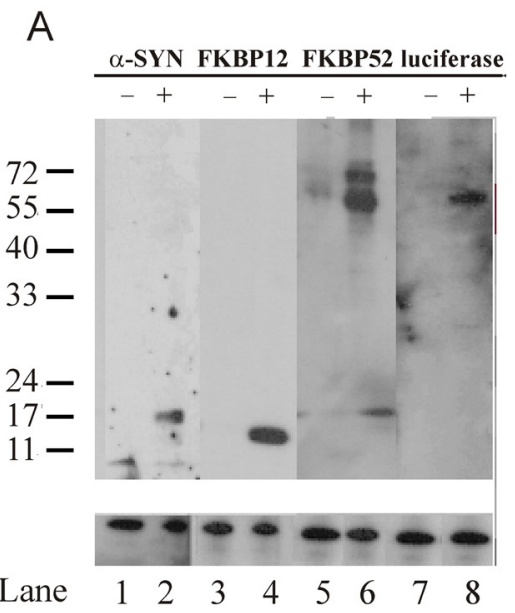

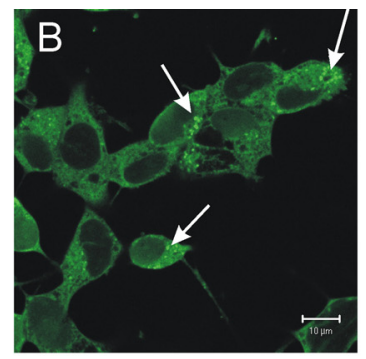
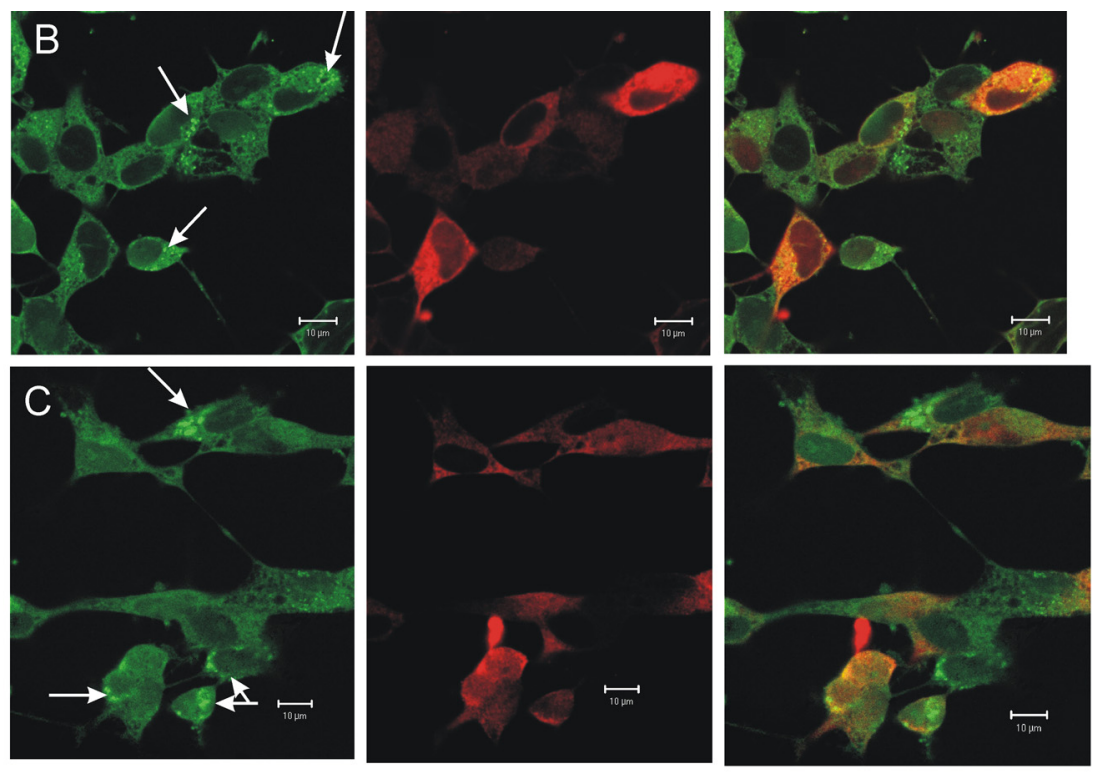

E
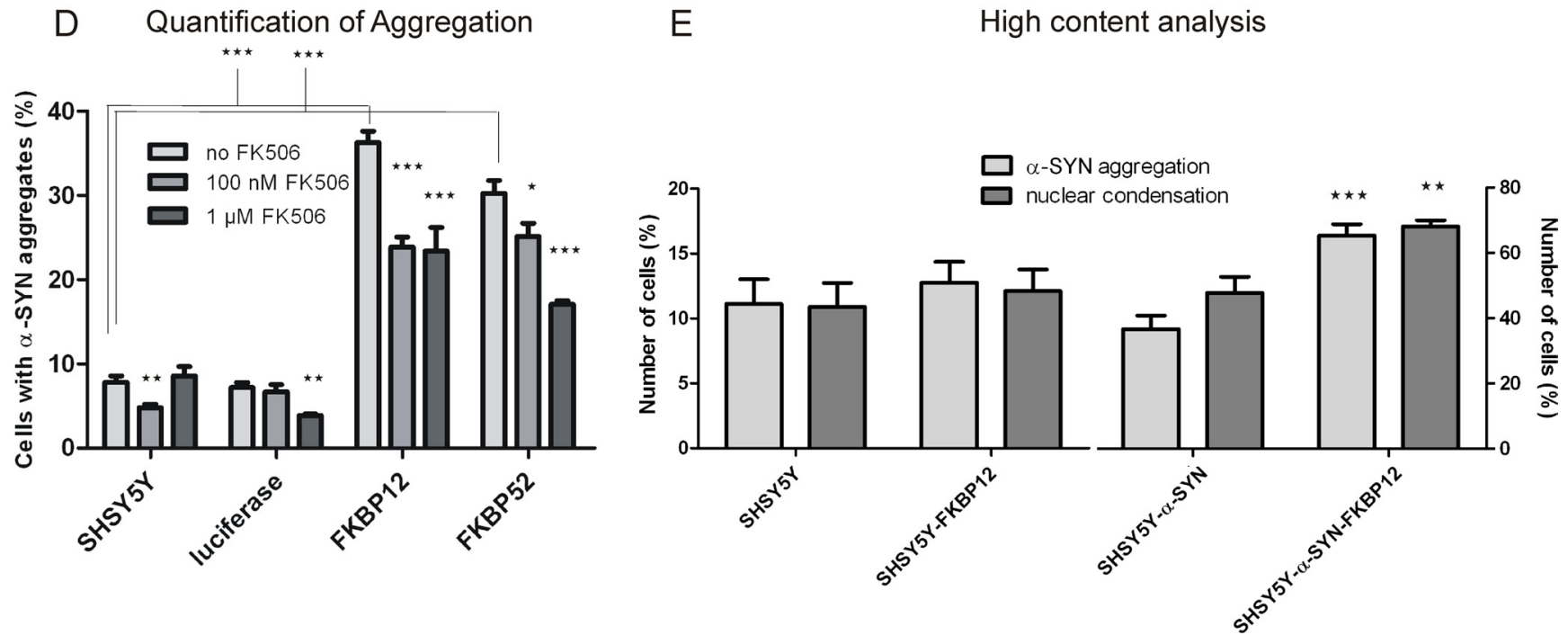

Figure 5. FKBP overexpression enhances $\alpha$-SYN aggregation and apoptosis in SHSY5Y cells. $\boldsymbol{A}$, Expression of $\alpha$-SYN, FKBP12, FKBP52, and luciferase in stable cell lines. Western blot of cell extracts of the different stable cell lines generated. Lanes 1,3,5, 7, Untransduced SHSY5Y cells. Lane 2, SHSY5Y cells overexpressing $\alpha$-SYN (14.4 kDa). Lane 4, SHSY5Y cells overexpressing FKBP12 (12 kDa). Lane 6, SHSY5Y cells overexpressing FKBP52 (52 kDa). Lane 8, SHSY5Y cells overexpressing luciferase (61 kDa). $\alpha$-Tubulin expression is shown below to confirm equal loading. $\boldsymbol{B}$, Subcellular localization of FKBP12 and $\alpha$-SYN aggregates in overexpression cell lines. Fixed and stained SHSY5Y cells overexpressing FKBP12 exposed to oxidative stress for $48 \mathrm{~h}$. Left, ThioS (green) stains $\alpha$-SYN fibrils (arrows). Middle, Immunocytochemical FKBP12 staining is shown in red. Right, Overlay image. C, Subcellular localization of FKBP52 and $\alpha$-SYN aggregates in overexpression cell lines. Fixed and stained SHSY5Y cells overexpressing FKBP52 exposed to oxidative stress for $48 \mathrm{~h}$. Left, ThioS (green) stains $\alpha$-SYN fibrils (arrows). Middle, Immunocytochemical FKBP52 staining is shown in red. Right, Overlap image. $\boldsymbol{D}$, Analysis of the effect of FKBP12 and FKBP52 overexpression on the aggregation of $\alpha$-synuclein. Cells were subjected to oxidative stress for $48 \mathrm{~h}$ in the presence or absence of FK506 before ThioS staining. WT SHSY5Y cells and luciferase-overexpressing cells are shown as negative controls. The percentage of ThioS-positive cells was determined in the different conditions. $p$ values are indicated compared with control condition (cell lines without FK506 treatment) or as indicated in the graph. $\boldsymbol{E}$, High-content analysis of the effect of FKBP12 overexpression on the aggregation of $\alpha$-SYN in both WT SHSY5Y and $\alpha$-SYN-SHSY5Y. The error bars show the SEM for each condition. $p$ values are indicated compared with control condition (cell lines with endogenous FKBP12 expression).

death. Furthermore, FKBP12 colocalized with $\alpha$-SYN aggregates in aged transgenic mice. Finally, long-term FK506 administration also significantly reduced $\alpha$-SYN aggregation and neuronal degeneration in vivo in a viral vector-induced mouse model for synucleinopathy.

A direct comparison of the manual versus the high-content method reveals that both can reliably quantify $\alpha$-synuclein aggregation in SHSY5Y cells. However, the latter has a higher sensitivity to detect $\alpha$-SYN aggregates (e.g., 55 vs $35 \%$ of positive cells in high-content vs manual method) (Fig. $1, F$ vs $E$ ). This is likely attributable to automatic detection of aggregates above a threshold of fluorescence in high-content analysis, whereas the manual method depends on a subjective estimation of aggregates by the blinded observer. Moreover, the manual analysis uses confocal images, including aggregates in one specific plane of the cell, whereas the high-content analysis counts all aggregates in the cell. This results in an underestimation of the percentage of positive cells with the manual method. Next to the higher sensitivity, other features make the high-content analysis the preferred method. It allows (1) an objective and fast quantification of a large number of cells and (2) quantification of several parameters at the same time in the same cells, resulting in detailed multiparametric information on individual cells in a large population. 

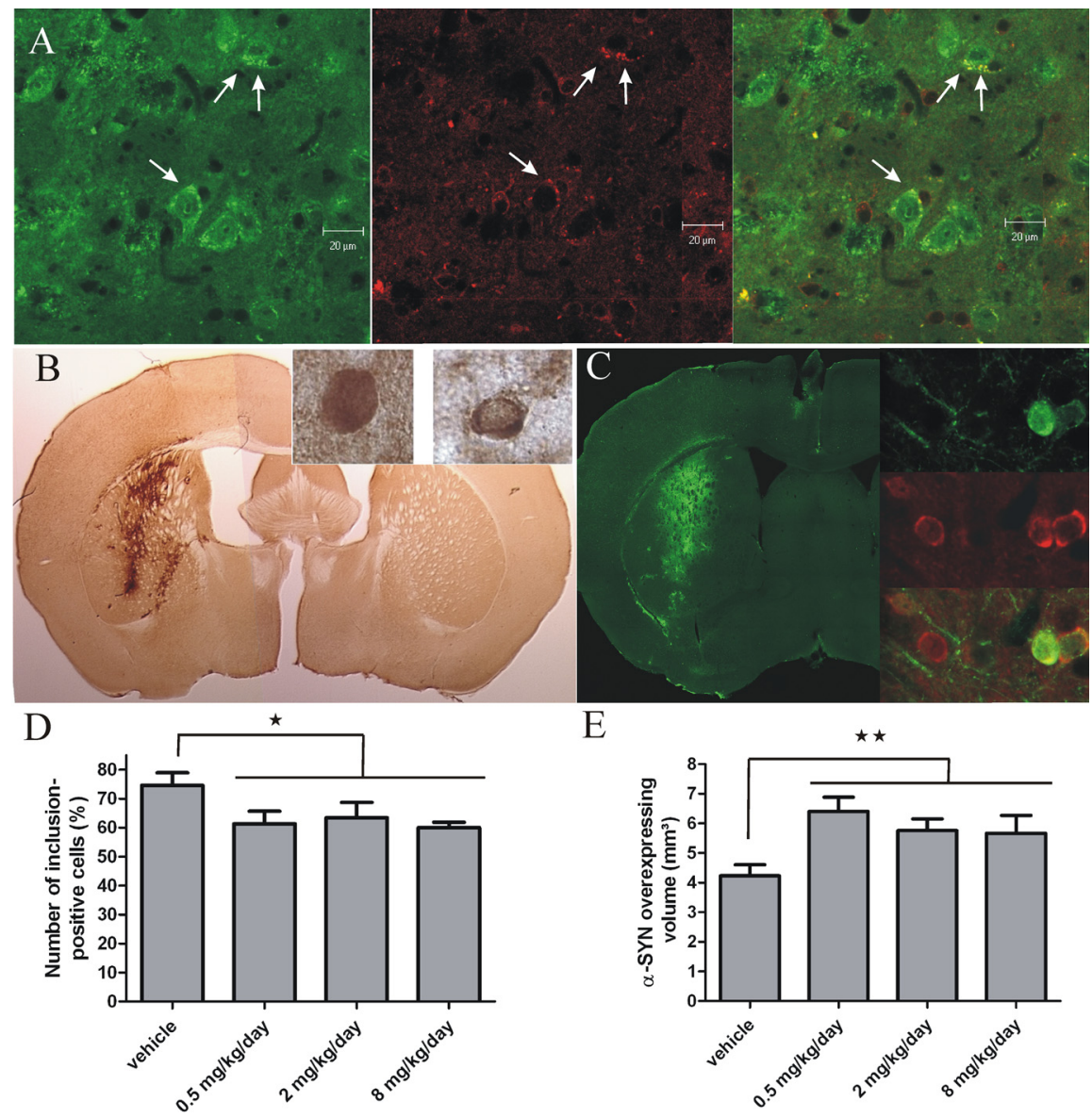

Figure 6. FK506 treatment reduces $\alpha$-SYN aggregation and neurodegeneration in mouse brain. $\boldsymbol{A}$, Colocalization of FKBP12 (red) and $\alpha$-SYN-positive LB-like inclusions (green) in the deep mesencephalic nuclei of 19-month-old Thy1-A30P- $\alpha$-SYN transgenic mice (Freichel et al., 2007). Cytoplasmic $\alpha$-SYN inclusions are indicated with arrows. $\boldsymbol{B}$, Immunohistochemical staining of $\alpha$-SYN in the striatum of mice injected with $\alpha$-SYN encoding LV. Inlay images show high-magnification views of two $\alpha$-SYNpositive neurons without (left) or with (right) $\alpha$-SYN inclusions. C, Immunofluorescent staining for $\alpha$-SYN and ubiquitin in the striatum 5 months after injection with $\alpha$-SYN LV. Detailed pictures on the right show colocalization of $\alpha$-SYN (green) and ubiquitin (red) in the inclusions. D, Number of $\alpha$-SYN aggregates in $\alpha$-SYN-overexpressing neurons in the striatum. The fraction of $\alpha$-SYNpositive neurons that contain inclusions was determined. FK506 treatment decreases the number of $\alpha$-SYN-positive neurons. Error bars show SEM ( $n=6-7)$. E, Survival of $\alpha$-SYN-overexpressing cells in the striatum after FK506 treatment. The $\alpha$-SYN-positive area was quantified using the Cavalieri method as a measure for the number of $\alpha$-SYN-overexpressing cells. In the groups treated with FK506, more $\alpha$-SYN-overexpressing cells survive. Error bars show the SEM ( $n=6-7)$.

For the mechanism of action, we provide strong evidence that FK506-mediated decreased $\alpha$-SYN aggregation is attributable to enzymatic inhibition of FKBP12 and possibly other FKBPs. We showed that FKBP12 accelerates recombinant $\alpha$-SYN aggregation in vitro via its enzymatic activity, an effect that could be efficiently blocked by FK506 (Gerard et al., 2008). Here we unambiguously document that the inhibition of $\alpha$-SYN aggregation by FK506 in neuronal cells can be reproduced by the RNA interference-mediated knockdown of FKBP12 or FKBP52. Moreover, overexpression of these enzymes enhanced $\alpha$-SYN aggregation, providing a direct link between FKBPs and $\alpha$-SYN aggregation. The weaker effect of FKBP52 versus FKBP12 suggests that FKBP52 interacts more weakly with $\alpha$-SYN or influences its aggregation in an indirect and less efficient way. Indeed, our recent data failed to detect an accelerating effect of recombinant FKBP52 on $\alpha$-SYN aggregation in vitro (A. Deleersnijder, $\mathrm{M}$. Gerard, Z. Debyser, V. Baekelandt, unpublished observations) Therefore, in light of a future therapy against PD, specific FKBP12 inhibitors seem most promising. Of note, the aggregate-accelerating effect of FKBP12 was more pronounced after overexpression of
$\alpha$-SYN, suggesting an increased activity of the enzyme in the presence of more substrate. Because $\alpha$-SYN levels remained constant during each experiment (data not shown), an effect of FKBP or FK506 on $\alpha$-SYN expression levels was ruled out.

The amino acid composition of $\alpha$-SYN is consistent with our hypothesis. The protein contains five prolines, all located in the $\mathrm{C}$ terminus. The C-terminal domain is (1) very accessible in most of the conformations observed in $\alpha$-SYN (Heise et al., 2005, 2008) and (2) an important regulatory domain in the aggregation of the protein (Hoyer et al., 2004). Important changes in this domain, such as a deletion (Hoyer et al., 2004), point mutation (Ulrih et al., 2008), or phosphorylation (Kragh et al., 2009), can expose the NAC domain to the solution, leading to hydrophobic interaction-driven aggregation. The prolines in the surface-accessible $\mathrm{C}$ terminus of $\alpha$-SYN constitute ideal substrates for PPIases, such as FKBP12. Indeed, the majority of the 5-6\% observed cis-peptide bonds in protein structures (Pal and Chakrabarti, 1999; Stewart et al., 1990) occur in surface-accessible bend, coil, or turn conformations (Pahlke et al., 2005). PPIases are known to act on prolines in these loop regions (Thali et al., 1994; Howard et al., 2003). The $\alpha$-SYN protein exists as an intrinsically disordered protein, known to adopt various conformations depending on interaction partner(s) and the environment. If PPIases induce certain folds in $\alpha$-SYN, the deregulation of their activity in conditions of oxidative stress might erroneously favor those conformations of $\alpha$-SYN that lead to aggregation and toxicity. It was shown previously that striatal FKBP12 mRNA and protein levels are both increased in an experimental 6-OHDA model of PD (Nilsson et al., 2007). Moreover, a higher FKBP12 expression was reported in the brain of PD patients. The protein was also found in Lewy bodies of surviving neurons (Avramut and Achim, 2002). Increased FKBP12 expression may consequently have a profound effect on the regulation of its activity.

Thanks to the multiparametric setup of the high-content assay, it can also be used to answer questions regarding the toxicity of $\alpha$-SYN. In literature, a strong causal link was already shown between $\alpha$-SYN (aggregation) and PD pathogenesis. Gene duplication or triplication of the WT $\alpha$-SYN gene is sufficient to induce a familial form of PD (Chartier-Harlin et al., 2004; Ibáñez et al., 2004). Overexpression of $\alpha$-SYN in animal models reproduces several features of Lewy-like pathology (Feany and Bender, 2000; Kirik et al., 2002; Lakso et al., 2003; Lauwers et al., 2003), but whether oligomeric or protofibrilar $\alpha$-SYN species are more toxic than the mature fibrils is still under debate (Volles et al., 2001; Ding et al., 2002; Volles and Lansbury, 2002). The multiparametric high-content analysis allowed us to measure $\alpha$-SYN ag- 
gregation and cell death simultaneously. A positive correlation between $\alpha$-SYN aggregation and apoptosis was measured by nucleus condensation and fragmentation, although we could not distinguish between $\alpha$-SYN oligomers, protofibrils, or mature fibrils as toxic species in the experimental setup. Nevertheless, this high-content cellular assay should be instrumental not only for additional research on $\alpha$-SYN and its aggregates but also to test new compounds targeting either $\alpha$-SYN aggregation or $\alpha$-SYN toxicity.

We focused our study on FKBP12 and FKBP52, although four members of the FKBP family are known to be highly expressed in the human brain (FKBP12, FKBP38, FKBP52, and FKBP65) (Steiner et al., 1992; Charters et al., 1994a,b; Coss et al., 1998). FKBP12 and FKBP52 were selected for two reasons. First, they were already linked to neuroregeneration in different reports (Gold et al., 1999; Kato et al., 2000; Brecht et al., 2003; Labrande et al., 2006), although the exact role of these proteins in (regenerating) neurons has not been resolved yet. Second, FKBP38 and FKBP65 seem less likely candidates for interaction with $\alpha$-SYN based on their localization in, respectively, mitochondria (Shirane and Nakayama, 2003) or endoplasmic reticulum (Patterson et al., 2000). Because the majority of cellular $\alpha$-SYN localizes to the cytoplasm and the aggregates are found exclusively cytoplasmic and neuritic, a causal link between FKBP38 or FKBP65 and $\alpha$-SYN aggregation is less likely. Nevertheless, mitochondrial localization of $\alpha$-SYN was recently reported, and a specific FKBP38 inhibitor also shows neuroregenerative properties (Edlich et al., 2006).

Based on our results, we put FKBPs forward as novel drug targets for causative treatment of PD mediating the inhibition of $\alpha$-SYN aggregation. Because FKBP ligands are clinically approved drugs, drug development looks straightforward.

The therapeutic effect of FK506 in our $\alpha$-SYN aggregation mouse model strongly supports the validity of our proposal. Still, at this stage, we cannot completely rule out that indirect effects of FK506, such as immune suppression, explain the observed phenotype. Viral vector-mediated overexpression or knockdown of FKBP12 in mouse models should allow clarification.

Efforts have been undertaken to develop non-immunosuppressive analogs of FK506 (non-immunosuppressive immunophilin ligands; NI-IL) (Pong and Zaleska, 2003; Poulter et al., 2004; Edlich et al., 2006; Sheehan et al., 2006; Liu et al., 2007). Of note, based on the neuroregenerative and neuroprotective properties of FKBP inhibitors, phase II clinical trials were recently performed with NI-IL. Neither in a 6 month nor in a 2 year trial with GPI-1485, a nonimmunosuppressive analog of FK506, improvements were seen in motor symptoms or DA neurotransmitter levels of patients with mild to moderate PD (Poulter et al., 2004; Sommer and Stacy, 2008). Several explanations are possible. First, GPI-1485 was not selected based on inhibitory potency against $\alpha$-SYN aggregation. Second, the selection of patients may have been more appropriate for a drugpromoting neuroregeneration than for a drug-preventing earlystage $\alpha$-SYN aggregation. Therefore, early-stage patients diagnosed with sensitive new techniques (Gasser, 2009; Tolosa et al., 2009) could profit from this therapeutic strategy. Interestingly, FKBP12specific knockdown in neuronal cells resulted in a much larger decrease in $\alpha$-SYN aggregation than addition of FK506 (Figs. 4, 5). Nonspecific inhibition of stress-induced FKBPs other than FKBP12 may counteract the neuroprotective inhibition of FKBP12. Additional research is required to answer these open questions. However, we believe it to be worthwhile revisiting the data from the clinical trials in light of the results of the present study.
In conclusion, we have shown that proteins of the FKBP family enhance aggregation of $\alpha$-SYN in neuronal cell culture and mouse brain. FK506 prevents $\alpha$-SYN aggregation and neuronal cell death by inhibition of FKBPs. These observations validate FKBPs as novel drug targets for PD.

\section{References}

Avramut M, Achim CL (2002) Immunophilins and their ligands: insights into survival and growth of human neurons. Physiol Behav 77:463-468.

Avramut M, Zeevi A, Achim CL (2001) The immunosuppressant drug FK506 is a potent trophic agent for human fetal neurons. Brain Res Dev Brain Res 132:151-157.

Bertoncini CW, Jung YS, Fernandez CO, Hoyer W, Griesinger C, Jovin TM, Zweckstetter M (2005) Release of long-range tertiary interactions potentiates aggregation of natively unstructured alpha-synuclein. Proc Natl Acad Sci U S A 102:1430-1435.

Brecht S, Schwarze K, Waetzig V, Christner C, Heiland S, Fischer G, Sartor K, Herdegen T (2003) Changes in peptidyl-prolyl cis/trans isomerase activity and FK506 binding protein expression following neuroprotection by FK506 in the ischemic rat brain. Neuroscience 120:1037-1048.

Charters AR, Kobayashi M, Butcher SP (1994a) Immunochemical analysis of FK506 binding proteins in neuronal cell lines and rat brain. Biochem Soc Trans 22:411S.

Charters AR, Kobayashi M, Butcher SP (1994b) The subcellular distribution of FK506 binding proteins in rat brain. Biochem Soc Trans 22:412S.

Chartier-Harlin MC, Kachergus J, Roumier C, Mouroux V, Douay X, Lincoln S, Levecque C, Larvor L, Andrieux J, Hulihan M, Waucquier N, Defebvre L, Amouyel P, Farrer M, Destée A (2004) Alpha-synuclein locus duplication as a cause of familial Parkinson's disease. Lancet 364:1167-1169.

Coss MC, Stephens RM, Morrison DK, Winterstein D, Smith LM, Simek SL (1998) The immunophilin FKBP65 forms an association with the serine/ threonine kinase c-Raf-1. Cell Growth Differ 9:41-48.

Davies TH, Ning YM, Sánchez ER (2005) Differential control of glucocorticoid receptor hormone-binding function by tetratricopeptide repeat (TPR) proteins and the immunosuppressive ligand FK506. Biochemistry 44:2030-2038.

Ding TT, Lee SJ, Rochet JC, Lansbury PT Jr (2002) Annular alpha-synuclein protofibrils are produced when spherical protofibrils are incubated in solution or bound to brain-derived membranes. Biochemistry 41:10209-10217.

Edlich F, Weiwad M, Wildemann D, Jarczowski F, Kilka S, Moutty MC, Jahreis G, Lücke C, Schmidt W, Striggow F, Fischer G (2006) The specific FKBP38 inhibitor $N$ - $\left(N^{\prime}, N^{\prime}\right.$-dimethylcarboxamidomethyl $)$ cycloheximide has potent neuroprotective and neurotrophic properties in brain ischemia. J Biol Chem 281:14961-14970.

Feany MB, Bender WW (2000) A Drosophila model of Parkinson's disease. Nature 404:394-398.

Freichel C, Neumann M, Ballard T, Müller V, Woolley M, Ozmen L, Borroni E, Kretzschmar HA, Haass C, Spooren W, Kahle PJ (2007) Agedependent cognitive decline and amygdala pathology in alpha-synuclein transgenic mice. Neurobiol Aging 28:1421-1435.

Galat A (2003) Peptidylprolyl cis/trans isomerases (immunophilins): biological diversity-targets-functions. Curr Top Med Chem 3:1315-1347.

Gasser T (2009) Genomic and proteomic biomarkers for Parkinson disease. Neurology 72:S27-S31

Geraerts M, Michiels M, Baekelandt V, Debyser Z, Gijsbers R (2005) Upscaling of lentiviral vector production by tangential flow filtration. J Gene Med 7:1299-1310.

Gerard M, Debyser Z, Desender L, Kahle PJ, Baert J, Baekelandt V, Engelborghs Y (2006) The aggregation of alpha-synuclein is stimulated by FK506 binding proteins as shown by fluorescence correlation spectroscopy. FASEB J 20:524-526.

Gerard M, Debyser Z, Desender L, Baert J, Brandt I, Baekelandt V, Engelborghs Y (2008) FK506 binding protein 12 differentially accelerates fibril formation of wild type alpha-synuclein and its clinical mutants A30P or A53T. J Neurochem 106:121-133.

Gold BG, Zeleny-Pooley M, Wang MS, Chaturvedi P, Armistead DM (1997) A nonimmunosuppressant FKBP-12 ligand increases nerve regeneration. Exp Neurol 147:269-278.

Gold BG, Densmore V, Shou W, Matzuk MM, Gordon HS (1999) Immunophilin FK506-binding protein 52 (not FK506-binding protein 12) me- 
diates the neurotrophic action of FK506. J Pharmacol Exp Ther 289: $1202-1210$.

Gold BG, Udina E, Bourdette D, Navarro X (2004) Neuroregenerative and neuroprotective actions of neuroimmunophilin compounds in traumatic and inflammatory neuropathies. Neurol Res 26:371-380.

Göthel SF, Marahiel MA (1999) Peptidyl-prolyl cis-trans isomerases, a superfamily of ubiquitous folding catalysts. Cell Mol Life Sci 55:423-436.

Guo X, Dawson VL, Dawson TM (2001) Neuroimmunophilin ligands exert neuroregeneration and neuroprotection in midbrain dopaminergic neurons. Eur J Neurosci 13:1683-1693.

Heise H, Hoyer W, Becker S, Andronesi OC, Riedel D, Baldus M (2005) Molecular-level secondary structure, polymorphism, and dynamics of full-length alpha-synuclein fibrils studied by solid-state NMR. Proc Natl Acad Sci U S A 102:15871-15876.

Heise H, Celej MS, Becker S, Riedel D, Pelah A, Kumar A, Jovin TM, Baldus M (2008) Solid-state NMR reveals structural differences between fibrils of wild-type and disease-related A53T mutant $\alpha$-Synuclein. J Mol Biol 380:444-450.

Howard BR, Vajdos FF, Li S, Sundquist WI, Hill CP (2003) Structural insights into the catalytic mechanism of cyclophilin A. Nat Struct Biol 10:475-481.

Hoyer W, Cherny D, Subramaniam V, Jovin TM (2004) Impact of the acidic C-terminal region comprising amino acids 109-140 on alpha-synuclein aggregation in vitro. Biochemistry 43:16233-16242.

Ibáñez P, Bonnet AM, Débarges B, Lohmann E, Tison F, Pollak P, Agid Y, Dürr A, Brice A (2004) Causal relation between alpha-synuclein gene duplication and familial Parkinson's disease. Lancet 364:1169-1171.

Kato H, Oikawa T, Otsuka K, Takahashi A, Itoyama Y (2000) Postischemic changes in the immunophilin FKBP12 in the rat brain. Brain Res Mol Brain Res 84:58-66.

Kirik D, Rosenblad C, Burger C, Lundberg C, Johansen TE, Muzyczka N, Mandel RJ, Björklund A (2002) Parkinson-like neurodegeneration induced by targeted overexpression of alpha-synuclein in the nigrostriatal system. J Neurosci 22:2780-2791.

Kragh CL, Lund LB, Febbraro F, Hansen HD, Gai WP, El-Agnaf O, RichterLandsberg C, Jensen PH (2009) $\alpha$-Synuclein aggregation and Ser-129 phosphorylation-dependent cell death in oligodendroglial cells. J Biol Chem 284:10211-10222.

Labrande C, Velly L, Canolle B, Guillet B, Masmejean F, Nieoullon A, Pisano P (2006) Neuroprotective effects of tacrolimus (FK506) in a model of ischemic cortical cell cultures: role of glutamate uptake and FK506 binding protein $12 \mathrm{kDa}$. Neuroscience 137:231-239.

Lakso M, Vartiainen S, Moilanen AM, Sirvi ö J, Thomas JH, Nass R, Blakely RD, Wong G (2003) Dopaminergic neuronal loss and motor deficits in Caenorhabditis elegans overexpressing human alpha-synuclein. J Neurochem 86:165-172.

Lauwers E, Debyser Z, Van Dorpe J, De Strooper B, Nuttin B, Baekelandt V (2003) Neuropathology and neurodegeneration in rodent brain induced by lentiviral vector-mediated overexpression of alpha-synuclein. Brain Pathol 13:364-372.

Lauwers E, Bequé D, Van Laere K, Nuyts J, Bormans G, Mortelmans L, Casteels C, Vercammen L, Bockstael O, Nuttin B, Debyser Z, Baekelandt $\mathrm{V}$ (2007) Non-invasive imaging of neuropathology in a rat model of alpha-synuclein overexpression. Neurobiol Aging 28:248-257.

Liu D, McIlvain HB, Fennell M, Dunlop J, Wood A, Zaleska MM, Graziani EI, Pong K (2007) Screening of immunophilin ligands by quantitative analysis of neurofilament expression and neurite outgrowth in cultured neurons and cells. J Neurosci Methods 163:310-320.

Murakami Y, Takamatsu H, Noda A, Osoda K, Ichise R, Tatsumi M, Tabata K, Sawamoto T, Nishimura S (2004) Pharmacokinetic animal PET study of FK506 as a potent neuroprotective agent. J Nucl Med 45:1946-1949.

Nilsson A, Sköld K, Sjögren B, Svensson M, Pierson J, Zhang X, Caprioli RM, Buijs J, Persson B, Svenningsson P, Andrén PE (2007) Increased striatal mRNA and protein levels of the immunophilin FKBP-12 in experimental Parkinson's disease and identification of FKBP-12-binding proteins. J Proteome Res 6:3952-3961.

Ostrerova-Golts N, Petrucelli L, Hardy J, Lee JM, Farer M, Wolozin B (2000) The A53T alpha-synuclein mutation increases iron-dependent aggregation and toxicity. J Neurosci 20:6048-6054.

Pahlke D, Freund C, Leitner D, Labudde D (2005) Statistically significant dependence of the Xaa-Pro peptide bond conformation on secondary structure and amino acid sequence. BMC Struct Biol 5:8.

Pal D, Chakrabarti P (1999) Cis peptide bonds in proteins: residues involved, their conformations, interactions and locations. J Mol Biol 294:271-288.

Patterson CE, Schaub T, Coleman EJ, Davis EC (2000) Developmental regulation of FKBP65. An ER-localized extracellular matrix binding-protein. Mol Biol Cell 11:3925-3935.

Pong K, Zaleska MM (2003) Therapeutic implications for immunophilin ligands in the treatment of neurodegenerative diseases. Curr Drug Targets CNS Neurol Disord 2:349-356.

Poulter MO, Payne KB, Steiner JP (2004) Neuroimmunophilins: a novel drug therapy for the reversal of neurodegenerative disease? Neuroscience 128:1-6.

Rulten SL, Kinloch RA, Tateossian H, Robinson C, Gettins L, Kay JE (2006) The human FK506-binding proteins: characterization of human FKBP19. Mamm Genome 17:322-331.

Sakai M, Hobara N, Hokama N, Kameya H, Ohshiro S, Sakanashi M, Saitoh H (2004) Increased bioavailability of tacrolimus after rectal administration in rats. Biol Pharm Bull 27:1480-1482.

Sheehan J, Eischeid A, Saunders R, Pouratian N (2006) Potentiation of neurite outgrowth and reduction of apoptosis by immunosuppressive agents: implications for neuronal injury and transplantation. Neurosurg Focus 20:E9.

Shirane M, Nakayama KI (2003) Inherent calcineurin inhibitor FKBP38 targets Bcl-2 to mitochondria and inhibits apoptosis. Nat Cell Biol 5:28-37.

Sommer DB, Stacy MA (2008) What's in the pipeline for the treatment of Parkinson's disease? Expert Rev Neurother 8:1829-1839.

Steiner JP, Dawson TM, Fotuhi M, Glatt CE, Snowman AM, Cohen N, Snyder SH (1992) High brain densities of the immunophilin FKBP colocalized with calcineurin. Nature 358:584-587.

Steiner JP, Hamilton GS, Ross DT, Valentine HL, Guo H, Connolly MA, Liang S, Ramsey C, Li JH, Huang W, Howorth P, Soni R, Fuller M, Sauer H, Nowotnik AC, Suzdak PD (1997) Neurotrophic immunophilin ligands stimulate structural and functional recovery in neurodegenerative animal models. Proc Natl Acad Sci U S A 94:2019-2024.

Stewart DE, Sarkar A, Wampler JE (1990) Occurrence and role of cis peptide bonds in protein structures. J Mol Biol 214:253-260.

Surguchov A (2008) Molecular cell biology of synucleins. In: International review of cell and molecular biology, Chap 6 (Kwang WJ, ed), pp 225-317. San Diego: Academic.

Taymans JM, Vandenberghe LH, Haute CV, Thiry I, Deroose CM, Mortelmans L, Wilson JM, Debyser Z, Baekelandt V (2007) Comparative analysis of adeno-associated viral vector serotypes $1,2,5,7$, and 8 in mouse brain. Hum Gene Ther 18:195-206.

Thali M, Bukovsky A, Kondo E, Rosenwirth B, Walsh CT, Sodroski J, Göttlinger HG (1994) Functional association of cyclophilin A with HIV-1 virions. Nature 372:363-365.

Tolosa E, Gaig C, Santamaría J, Compta Y (2009) Diagnosis and the premotor phase of Parkinson disease. Neurology 72:S12-S20.

Ulrih NP, Barry CH, Fink AL (2008) Impact of Tyr to Ala mutations on $\alpha$-synuclein fibrillation and structural properties. Biochim Biophys Acta 1782:581-585.

Van den Haute C, Eggermont K, Nuttin B, Debyser Z, Baekelandt V (2003) Lentiviral vector-mediated delivery of short hairpin RNA results in persistent knockdown of gene expression in mouse brain. Hum Gene Ther 14:1799-1807.

Volles MJ, Lee SJ, Rochet JC, Shtilerman MD, Ding TT, Kessler JC, Lansbury PT Jr (2001) Vesicle permeabilization by protofibrillar alpha-synuclein: implications for the pathogenesis and treatment of Parkinson's disease. Biochemistry 40:7812-7819.

Volles MJ, Lansbury PT Jr (2002) Vesicle permeabilization by protofibrillar alpha-synuclein is sensitive to Parkinson's disease-linked mutations and occurs by a pore-like mechanism. Biochemistry 41:4595-4602.

Woods WS, Boettcher JM, Zhou DH, Kloepper KD, Hartman KL, Ladror DT, Qi Z, Rienstra CM, George JM (2007) Conformation-specific binding of alpha-synuclein to novel protein partners detected by phage display and NMR spectroscopy. J Biol Chem 282:34555-34567. 